\title{
A study of 24 cases of salivary gland carcinoma with distant metastasis
}

\author{
TAKASHI MASUI ${ }^{1}$, HIROKAZU UEMURA ${ }^{1}$, ICHIRO OTA $^{1}$, TAKAHIRO KIMURA ${ }^{1}$, \\ DAISUKE NISHIKAWA ${ }^{2}$, TOSHIAKI YAMANAKA ${ }^{1}$, KATSUNARI YANE $^{2}$ and TADASHI KITAHARA ${ }^{1}$ \\ ${ }^{1}$ Department of Otolaryngology-Head and Neck Surgery, Nara Medical University, Kashihara, Nara 634-8522; \\ ${ }^{2}$ Department of Otolaryngology, Kindai University School of Medicine, Nara Hospital, Ikoma, Nara 630-0293, Japan
}

Received March 5, 2021; Accepted May 26, 2021

DOI: $10.3892 / \operatorname{mco} .2021 .2345$

\begin{abstract}
Salivary gland carcinoma is a relatively rare disease of the head and neck. Although it frequently presents with distant metastases, few reports have been published on this subject. The present study investigated the prognosis of patients with distant metastases from salivary gland cancer. A total of 24 cases of salivary gland carcinoma with distant metastasis who were initially treated at the Department of Otolaryngology-Head and Neck Surgery of Nara Medical University during a 16-year period from August 2004 to July 2020 were included. The histopathological types included salivary duct carcinoma (8 cases), adenoid cystic carcinoma (6 cases), myoepithelial carcinoma (3 cases), Squamous cell carcinoma (2 cases), adenocarcinoma (2 cases), acinic cell carcinoma (2 cases) and mucoepidermoid carcinoma (1 case). A total of 18 patients had stage IV carcinoma, which represented the majority. Of all patients, $\sim 80 \%$ developed distant metastases within 2 years of initial diagnosis. Survival rates after the appearance of distant metastases were $43.5 \%$ at 5 years and $14.5 \%$ at 10 years. The results of the current study revealed that no factors significantly influenced long-term prognosis after the development of distant metastases. In future, it may be necessary to re-examine these results in a larger sample size and standardise treatment methods as a result.
\end{abstract}

\section{Introduction}

Carcinomas arising from salivary glands, such as the parotid, submandibular, sublingual, and minor salivary glands, are relatively uncommon (1-4). A large number of clinical statistics have been reported for salivary gland carcinoma (5-9). The

Correspondence to: Dr Takashi Masui, Department of Otolaryngology-Head and Neck Surgery, Nara Medical University, 840 Shijo-cho, Kashihara, Nara 634-8522, Japan

E-mail:masui@naramed-u.ac.jp

Key words: salivary gland carcinoma, distant metastasis, survival rate, prognostic factors treatment of salivary gland cancer generally involves surgery and postoperative radiotherapy $(5,6,10)$. However, these are often insufficient for high-grade disease, and recurrence or metastasis often occurs, leading to a poor prognosis $(4,11)$. The prognosis depends on the site of origin, histopathology, and staging $(1,12)$ of the carcinoma. However, the small number of cases and variety of pathological presentations make it difficult to study a coherent number of cases on a consistent basis. Furthermore, the treatment of patients with distant metastases, which are more difficult to treat, varies between centres. There have been few studies on distant metastases in salivary adenocarcinoma, and few reports on their treatment and prognosis. In the present study, we investigated the prognosis of patients with distant metastases from salivary gland carcinoma.

\section{Materials and methods}

Patient selection. The subjects included 24 patients with salivary gland carcinoma with distant metastasis, initially treated at the Department of Otolaryngology-Head and Neck Surgery, Nara Medical University, during a 16-year period from August 2004 to July 2020. The observation period ranged from 2 to 133 months (median 23.5 months).

Prognostic factors. We examined the time to distant metastasis, overall survival, overall survival based on the date of detection of distant metastasis, and differences in survival based on the date of distant metastasis detection according to sex, age, site of origin, number of metastatic organs, stage, and whether the metastasis was resectable.

Statistical analysis. Statistical analyses were performed using StatMate V statistical software (ATMS Co., Ltd.). Overall survival after salivary gland carcinoma diagnosis with distant metastasis was analysed using the Kaplan-Meier method, and the groups were compared using the log-rank test. Statistical significance was set at $\mathrm{P}<0.05$. A multivariable analysis was not performed because of the small number of patients.

\section{Results}

Clinical characteristics The patients' ages ranged from 45 to 84 years, with a median age of 68 years. The sample 
Table I. Characteristics of the patients with salivary gland carcinoma.

\begin{tabular}{lc}
\hline Characteristics & No. of patients $(\mathrm{n}=24)$ \\
\hline Sex & \\
Female & 16 \\
Male & $68(45-84)$ \\
Median age, years (range) & \\
Site of cancer origin & 16 \\
Parotid gland & 7 \\
Submandibular gland & 1 \\
Sublingual gland & \\
Stage & 6 \\
I-III & 18 \\
IV & \\
\hline
\end{tabular}

Table II. Histopathological type of salivary gland carcinoma.

\begin{tabular}{ll}
\hline Histopathological type & No. of patients $(\%)$ \\
\hline Salivary duct carcinoma & $8(33.3)$ \\
Adenoid cystic carcinoma & $6(25.0)$ \\
Myoepithelial carcinoma & $3(12.5)$ \\
Squamous cell carcinoma & $2(8.3)$ \\
Adenocarcinoma & $2(8.3)$ \\
Acinic cell carcinoma & $2(8.3)$ \\
Mucoepidermoid carcinoma & $1(4.2)$ \\
\hline
\end{tabular}

Table III. T and N classification of salivary gland carcinoma with distant metastasis.

\begin{tabular}{lcccc}
\hline & \multicolumn{4}{c}{ T grade } \\
\cline { 2 - 5 } N grade & T1 & T2 & T3 & T4a \\
\hline N0 & 0 & 4 & 1 & 4 \\
N1 & 0 & 1 & 0 & 0 \\
N2b & 1 & 2 & 3 & 6 \\
N3b & 0 & 0 & 1 & 1 \\
\hline
\end{tabular}

predominantly consisted of male patients, with 16 males and eight females. The parotid gland was involved in 16 cases, the submandibular gland in 7 cases, and the sublingual gland in 1 case. In terms of staging, the majority of the cases (18 out of 24) were stage IV (Table I). As shown in Table II, salivary duct carcinoma was the most common histological type, with 8 cases, adenoid cystic carcinoma in 6 cases, and other histological types with less than 3 cases such as myoepithelial carcinoma (3 cases), Squamous cell carcinoma (2 cases), Adenocarcinoma (2 cases), Acinic cell carcinoma ( 2 cases) and Mucoepidermoid carcinoma (1 case). Table III shows the TN classification. Stage IV
Table IV. Site of distant metastasis.

\begin{tabular}{lc}
\hline Site of distant metastasis & No. of patients $(\%)$ \\
\hline Lung & $20(83.3)$ \\
Bone & $5(20.8)$ \\
Mediastinum & $4(16.7)$ \\
Adrenal glands & $3(12.5)$ \\
Axilla & $2(8.3)$ \\
Brain & $2(8.3)$ \\
Liver & $1(4.2)$ \\
Skin & $1(4.2)$
\end{tabular}

Duplicates are present within this table.

Table V. Treatment of distant metastases.

\begin{tabular}{lc}
\hline Treatment type & Number of patients \\
\hline No treatment & 3 \\
Surgery & 6 \\
Chemotherapy & 17 \\
Docetaxel + cisplatin & 4 \\
Docetaxel & 5 \\
TS-1 & 9 \\
Trastuzumab + paclitaxel & 2 \\
Nivolumab & 2 \\
Radiotherapy & 6 \\
\hline
\end{tabular}

Duplicates are present within this table as patients received multiple treatments.

Table VI. Prognostic factors of salivary gland carcinoma with distant metastasis.

\begin{tabular}{lc}
\hline Factors & P-value \\
\hline Sex (female or male) & 0.172 \\
Age ( $\geq 70$ vs. <70 years) & 0.718 \\
Tumor location (parotid gland or & 0.229 \\
Submandibular gland) & \\
Number of organs with distant metastases ( $\geq 2$ vs. 1) & 0.317 \\
Stage (I-III or IV) & 0.369 \\
Radical resection against distant metastasis (+ or -) & 0.057
\end{tabular}

accounted for three quarters of the total. As shown in Table IV, the lung was the most common site of distant metastasis in 20 cases, followed by the bone, mediastinum, and adrenal gland. As shown in Table V, treatment of distant metastases included pneumonectomy (6 cases), radiotherapy (6 cases), chemotherapy (17 cases) which included docetaxel + cisplatin, docetaxel, TS-1, trastuzumab + paclitaxel, and nivolumab. Of the 17 patients who received 


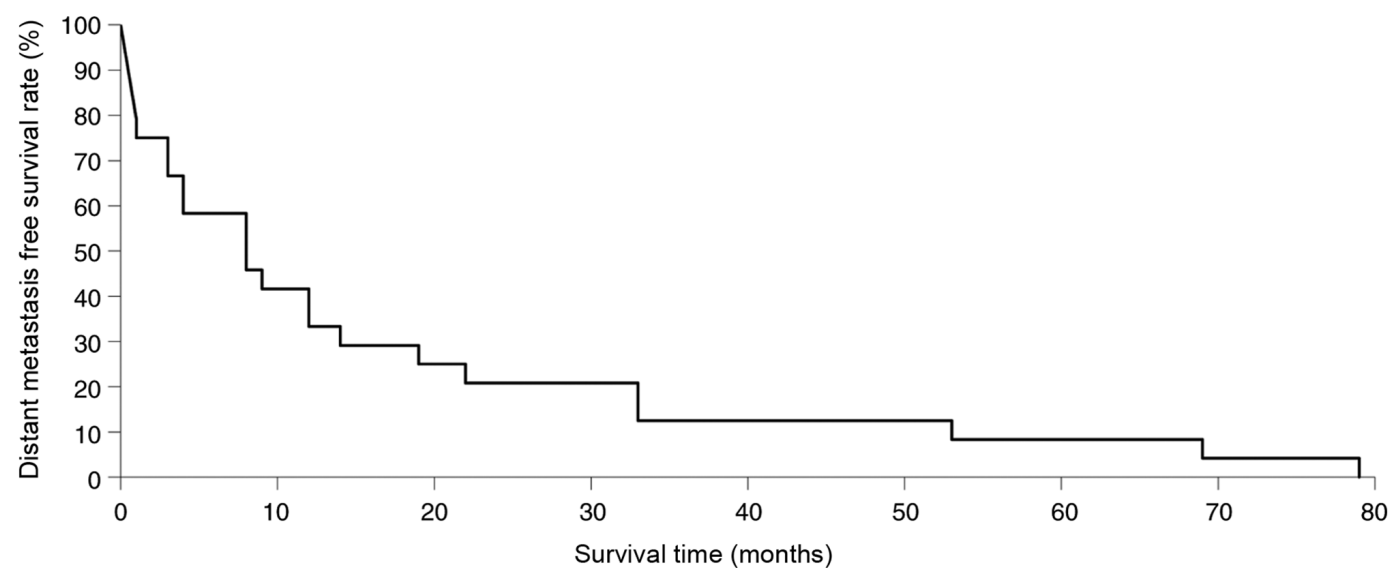

Figure 1. Kaplan-Meier analysis of distant metastases-free survival.

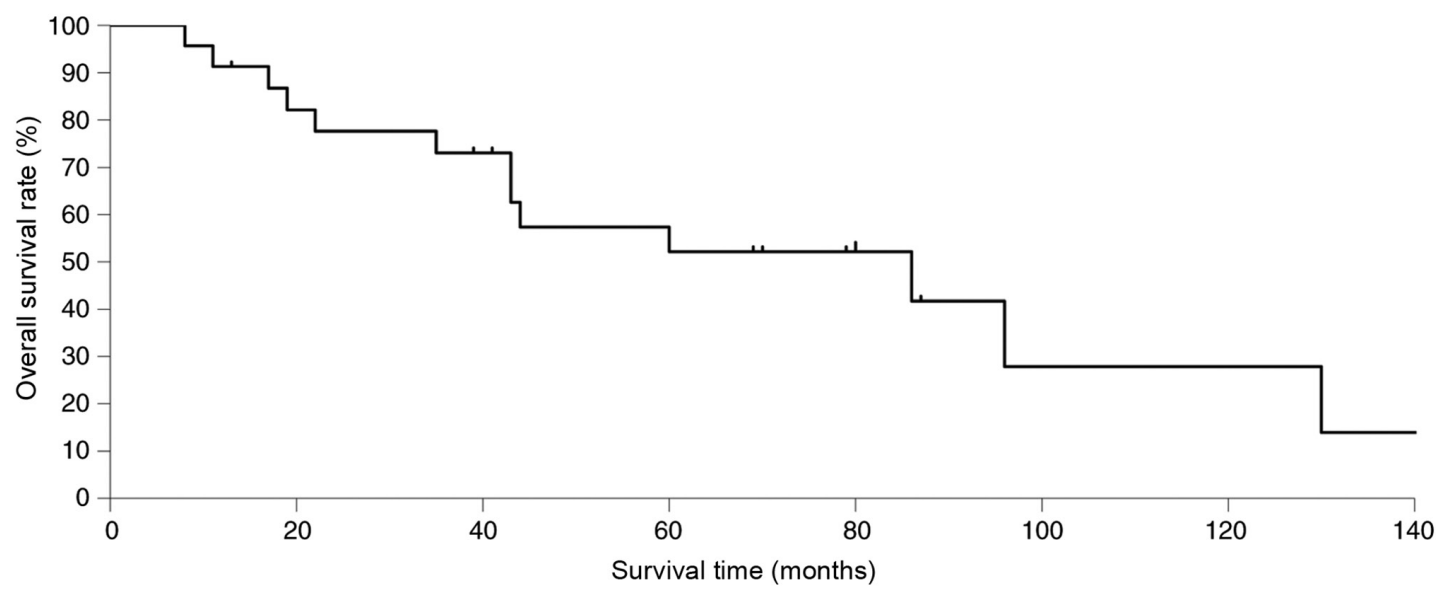

Figure 2. Kaplan-Meier analysis of overall survival from initial treatment.

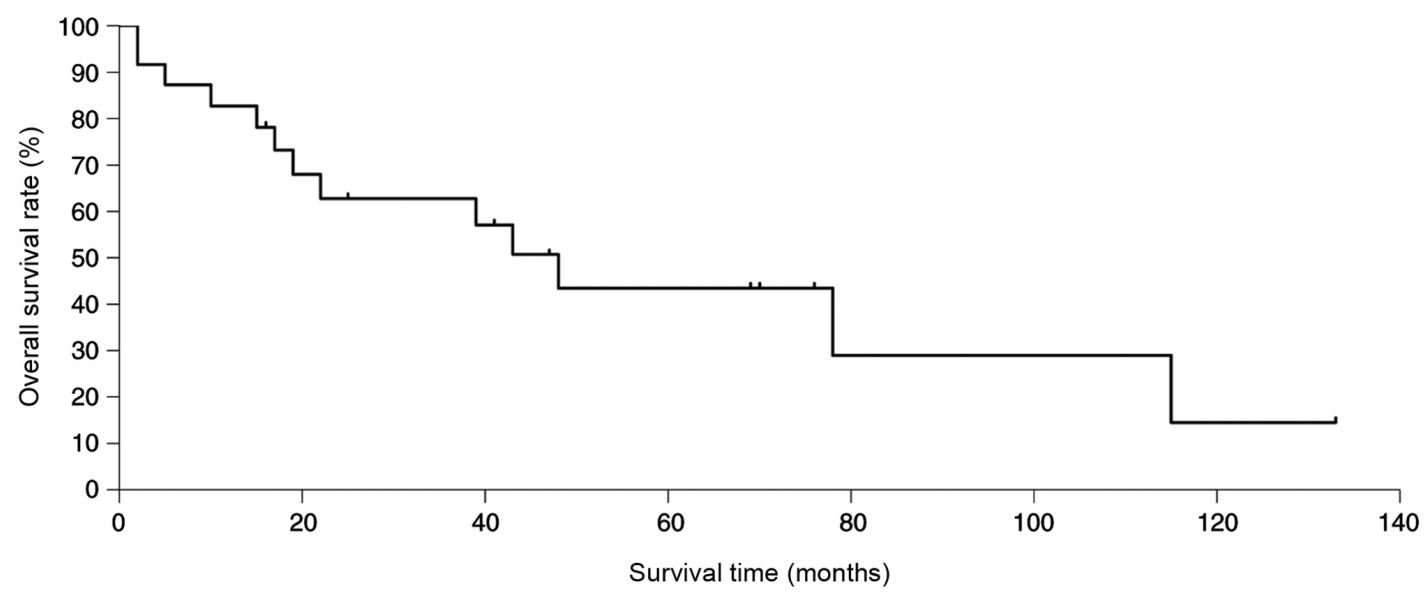

Figure 3. Kaplan-Meier analysis of overall survival after the appearance of distant metastases.

chemotherapy after the appearance of distant metastases, 5 survived: 2 with trastuzumab + paclitaxel and 1 each with docetaxel+cisplatin, TS-1 and nivolumab.

Prognostic factors. Approximately $80 \%$ of all patients developed distant metastases within 2 years of the initial diagnosis (Fig. 1). As shown in Fig. 2, the 5-year and 10-year survival rates after initial treatment were 52.2 and $27.8 \%$, respectively. After the appearance of distant metastases, the 5-year survival rates were $43.5 \%$ and the 10 -year survival rate was $14.5 \%$, respectively (Fig. 3). As shown in Table VI, no significant prognostic factors were found to significantly influence the long-term prognosis after the appearance of distant metastases among patients in this study. 


\section{Discussion}

The prognosis of salivary gland carcinoma with distant metastases is poor, and the variety of histopathological types often makes it difficult to find a well-established treatment. In the present study, unlike previous reports, salivary duct carcinoma, rather than adenocarcinoma or adenoid cystic carcinoma, was the most common type $(1,13,14)$. This may be because of our focus on cases with distant metastases. Similar to previous reports, we observed that the carcinoma occurred predominantly in males, and the parotid gland was the most common site of occurrence $(15,16)$.

In this study, the 5-year and 10-year survival rates after the appearance of distant metastases were 43.5 and $14.5 \%$, respectively. The 5-year survival rate is not low for head and neck cancer after the appearance of distant metastases, but the 10 -year survival rate is low at $14.5 \%$, which may a feature of salivary gland carcinoma. Even if the patients survive for more than 5 years, the mortality rate at 10 years would be $70 \%$, suggesting that long-term observation is necessary.

As shown in Table VI, sex was not predominantly associated with prognosis. No details available on hormone regulations of the salivary gland carcinogenesis. This is a subject for further study. There were no significant differences in survival according to sex, age, site of origin, number of metastatic organs, stage, or whether metastases were resectable. This means that once distant metastases develop, the prognosis is similar regardless of the number of metastatic organs or the stage of the disease. However, in other words, even if the disease has spread to multiple organs and the original stage is IV, there is still the possibility of long-term prognosis depending on treatment. In the present study, we were not able to conduct a comprehensive study because at present there is no unified treatment plan based on the histopathological type of the carcinoma, but we hope to study a larger number of cases and establish better treatment protocols in the future. The only difference was that patients with resectable metastases tended to have a longer survival than those with unresectable metastases $(\mathrm{P}=0.060)$. In other words, in patients with distant metastases that are resectable, resection should be performed whenever possible. Molecular-targeted therapy and immunotherapy are promising strategies for refractory carcinoma such as salivary gland carcinoma. Two patients treated with trastuzumab and one with nivolumab are alive, but the number of patients is still small and has not been fully investigated.

In this study, we investigated salivary gland carcinoma with distant metastasis. There are few reports focusing on distant metastases, and we believe that this is a valuable report in this regard. Although the treatment could not be studied in depth due to aforementioned reasons, we were able to evaluate the long-term prognosis and prognostic factors among the included patients in detail. In the future, studies with a larger number of cases should be performed, and the treatment protocols should be standardised.

\section{Acknowledgements}

Not applicable.

\section{Funding}

No funding was received.

\section{Availability of data and materials}

The datasets used and/or analyzed during the present study are available from the corresponding author on reasonable request.

\section{Authors' contributions}

TM, HU, IO and TKit conceived and designed the current study. TM, HU, IO, TKim, DN, TY, KY and TKit acquired the data. TM and IO confirmed the authenticity of all the raw data. TM, HU, IO, TKim and DN analyzed and interpreted the data. TM and TKit drafted the manuscript. TM, HU, IO and TKit reviewed and edited the manuscript. All authors have accepted their responsibility for the entire content of this manuscript and approved submission. All authors read and approved the final manuscript.

\section{Ethics approval and consent to participate}

The present study was approved by ethics committee of Nara Medical University Hospital (approval no. 2923). Written informed consent was obtained from the patients.

\section{Patients consent for publication}

Not applicable.

\section{Competing interests}

The authors declare that they have no competing interests.

\section{References}

1. Licitra L, Grandi C, Prott FJ, Schornagel JH, Bruzzi P and Molinari R: Major and minor salivary glands tumours. Crit Rev Oncol Hematol 45: 215-225, 2003.

2. Pinkston JA and Cole P: Incidence rates of salivary gland tumors: Results from a population-based study. Otolaryngol Head Neck Surg 120: 834-840, 1999.

3. Jones AV, Craig GT, Speight PM and Franklin CD: The range and demographics of salivary gland tumours diagnosed in a UK population. Oral Oncol 44: 407-417, 2008.

4. Bell RB, Dierks EJ, Homer L and Potter BE: Management and outcome of patients with malignant salivary gland tumors. J Oral Maxillofac Surg 63: 917-928, 2005.

5. Garden AS, el-Naggar AK, Morrison WH, Callender DL, Ang KK and Peters LJ: Postoperative radiotherapy for malignant tumors of the parotid gland. Int J Radiat Oncol Biol Phys 37: 79-85, 1997.

6. Gurney TA, Eisele DW, Weinberg V, Shin E and Lee N: Adenoid cystic carcinoma of the major salivary glands treated with surgery and radiation. Laryngoscope 115: 1278-1282, 2005.

7. Forrest J, Campbell P, Kreiger N and Sloan M: Salivary gland cancer: An exploratory analysis of dietary factors. Nutr Cancer 60: 469-473, 2008.

8. Ostman J, Anneroth G, Gustafsson H and Tavelin B: Malignant salivary gland tumours in Sweden 1960-1989-an epidemiological study. Oral Oncol 33: 169-176, 1997.

9. Silas OA, Echejoh GO, Manasseh AN and Mandong BM: Patterns of malignant salivary gland tumours in Jos university teaching hospital (JUTH), Jos: A ten-year retrospective study. Niger J Med 18: 282-285, 2009. 
10. Kaur J, Goyal S, Muzumder S, Bhasker S, Mohanti BK and Rath GK: Outcome of surgery and post-operative radiotherapy for major salivary gland carcinoma: Ten year experience from a single institute. Asian Pac J Cancer Prev 15: 8259-8263, 2014.

11. Koul R, Dubey A, Butler J, Cooke AL, Abdoh A and Nason R: Prognostic factors depicting disease-specific survival in parotid-gland tumors. Int J Radiat Oncol Biol Phys 68: 714-718, 2007.

12. Hocwald E, Korkmaz H, Yoo GH, Adsay V, Shibuya TY, Abrams J and Jacobs JR: Prognostic factors in major salivary gland cancer. Laryngoscope 111: 1434-1439, 2001.
13. Wahlberg P, Anderson H, Biörklund A, Möller T and Perfekt R: Carcinoma of the parotid and submandibular glands-a study of survival in 2465 patients. Oral Oncol 38: 706-713, 2002.

14. Zbären P, Guélat D, Loosli H and Stauffer E: Parotid tumors: Fine-needle aspiration and/or frozen section. Otolaryngol Head Neck Surg 139: 811-815, 2008.

15. Ito FA, Ito K, Vargas PA, de Almeida OP and Lopes MA: Salivary gland tumors in a Brazilian population: A retrospective study of 496 cases. Int J Oral Maxillofac Surg 34: 533-536, 2005.

16. Otoh EC, Johnson NW, Olasoji H, Danfillo IS and Adeleke OA: Salivary gland neoplasms in Maiduguri, north-eastern Nigeria. Oral Dis 11: 386-391, 2005. 